\title{
Immersive visual technologies and human health
}

\author{
Simone Grassini * \\ Department of Psychology, Norwegian University of \\ Science and Technology, Trondheim, Norway \\ simone.grassini@ntnu.no
}

\begin{abstract}
The fast-phased adoption of modern immersive visual technologies in many fields calls for an assessment of potential costs and benefits from the point of view of human health. Humans often reported feelings of discomfort in the experience of virtual reality (VR), however, it is still not known what may promote this uncomfortable symptomatology. In the framework of research on human-computer interaction, part of the discomfort derived from the use of immersive visual technologies has been attribute to technological factors. Among the most cited technological limitations is the vergence-accommodation conflict that human eyes are experiencing in displays delivering $3 \mathrm{D}$ images, and that have been connected to feelings of discomfort and tiredness. In the present investigation we attempt to briefly review the most important pieces of evidence presented in the literature, on the possible technological limitation of immersive visual technologies and its possible effects on human health and the way those effects were analyzed, and the ill symptomologies were assessed. Furthermore, possible solutions for reducing negative effects of the use of 3D media were shortly analyzed, and new perspective on the issue reviewed. Finally, we will attempt to assess the way immersive visual technology can be used in the medical field with the aim to improve human health.
\end{abstract}

\section{CCS CONCEPTS}

- Social and professional topics; • User characteristics;

\section{KEYWORDS}

Health, Users, Risks, Medical applications

\section{ACM Reference Format:}

Simone Grassini and Karin Laumann. 2021. Immersive visual technologies and human health. In European Conference on Cognitive Ergonomics 2021 (ECCE 2021), April 26-29, 2021, Siena, Italy. ACM, New York, NY, USA, 6 pages. https://doi.org/10.1145/3452853.3452856

\section{INTRODUCTION}

Experts used to worry that immersive visual technologies (IVTs) may have negative effect on human health, from its very early technological developments (see e.g. [1], [2]). The Holy Grail for 3D displays is to produce a scene that, to human eyes, is indistinguishable

\footnotetext{
${ }^{\dagger}$ Corresponding author

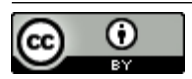

This work is licensed under a Creative Commons Attribution International 4.0 License.

ECCE 2021, April 26-29, 2021, Siena, Italy

(C) 2021 Copyright held by the owner/author(s).

ACM ISBN 978-1-4503-8757-6/21/04.

https://doi.org/10.1145/3452853.3452856
}

\author{
Karin Laumann \\ Department of Psychology, Norwegian University of \\ Science and Technology, Trondheim, Norway \\ karin.laumann@ntnu.no
}

from reality, and therefore reduces the problems related to human vision. To achieve the goal, the display would need to deceive all perceptual cues that the human visual system uses to sense the world. However, a number of virtual reality (VR) users complain to feel dizzy or nauseous after watching a 3D movie or using a virtual reality headset. Some users may be able to detect subconsciously the subtle differences between the virtual 3D scene presented to them and the real world, causing them a sense of disorientation. The human visual system perceives depth with multiple cues. When humans observe the world normally, the eye-muscles first point the eyeballs toward the visual target-vergence-and then focuses the lenses-accommodation-, after which these two processes are coupled to create a coherent picture [3]. However, these complex physiological processes may not work optimally in a computerized environment, and therefore cause a sense of discomfort in the user. Psychophysical research (see e.g. [4]-[9]) and user-experience research [10]-[12] have suggested that the perceived 3D structure will differ between the real and the computer-generated VR scene. Physiological brain activity in perceiving real-world information and in VR may be different. In an animal study, neurons in the brain region associated with spatial learning were shown to behave differently in virtual environments compared to in real ones, with a large number of neurons shutting down while in VR [13]. These findings highlight the need for more research on the long-term effects of VR, especially on the developing brain [14]. In the last few years, VR technology is gaining widespread use also outside the nice of tech enthusiasts and the field of entertainment. Companies and organizations are adopting the technology, aiming for example, to reduce training costs, or improve safety [15]. The wider adoption of the technology also calls for investigations on the possible health effects that these technologies may promote in the users. Please note that the present article, the terminologies "IVT(s)" and "VR" are used as synonyms, and identify any type of computer-generated experience, provided by hardware/software able to promote a high level of sense of presence in the user. The present study is a literature review. The studies here reported and discussed were included based on the prior knowledge of the authors regarding recent development on the topic. This study aims to: 1) review the string of evidence on the ill effects of the use of IVTs, and discuss methods of assessment and theories, 2) discuss the technical limitations of the technology, as well as possible solutions and developments, and 3) asses the possible benefits of the use of IVTs in the clinical field.

\section{DISCOMFORT DURING THE VIRTUAL EXPERIENCE}

The symptoms of discomfort during VR simulation, or in the period following it, are essentially expressed in cybersickness (CS). 
This is a constellation of symptoms of discomfort and malaise produced by VR exposure. CS is typically categorized as a form of visually induced motion sickness (VIMS), which describes any sickness produced by observation of visual motion, and it is distinct but symptomatically similar to - simulator sickness (SS), which is the terminology that has been used for the sickness produced by vehicle simulators. A slight distinction has been made between the experience of CS and SS; while CS is characterized by a prevalence of disorientation symptoms, SS appears to be predominated by oculomotor symptoms [16]. While many individuals experience SS in VR, others appear to be robust to the symptoms. Even if some of the theorists may disagree (see [16]), many of the published studies have referred to CS and SS as synonyms, especially in recent years (see e.g. [17]-[19]). For simplicity, in the present article, the phenomenon will be hereinafter referred as "SS". The most common approach for measuring SS involves subjective measures, particularly multi-item questionnaires such as the Simulator Sickness Questionnaire (SSQ; [20]) which includes 16 items (e.g., eyestrain, dizziness, and headache) on a four-point scale (none, slight, moderate, or severe). Common practice with the SSQ is to generate a total sickness score as well as scores for each subscale of oculomotor discomfort, disorientation, and nausea. A shortened version of the SSQ (Short Symptoms Checklist, SSC; [21]), consisting of two items from each subscale, has been developed and employed in a small number of studies [21]. Given the dynamic nature of SS, which tends to increase during VR exposure and slowly dissipate following VR termination, there are clear challenges involved in using one-shot questionnaire measurements to evaluate SS [20]. Single item scales for measuring SS have also been developed and validated, providing an efficient method for assessing the temporal evolution of SS (e.g., Fast Motion Sickness Scale; [22]. Recent development in the field have proposed the use of psychophysiological correlates as a way to estimate the level of SS [23], [24]. The study of Dennison, Zachary, and D'Zmura [25] found that objective physiological rate of stomach activity, blinking and breathing rate, are good predictors for establishing the level of SS of a user. In another study [26], ,SS during the use of HMDs was found to correlate to a decrease in heart-rate (which the authors proposed to be connected to the phenomenon of "preparation for action"), inversely correlated with blink-rate and positively correlated with changes in pupil diameters during the VR exposure. The authors also proposed the development of a real-time discomfort detection algorithm based on physiological parameters for the evaluation of SS discomfort during VR experience. Physiological indexes may be used in the future to monitor in a continuous and objective way the onset and development of SS during the use of IVTs. However, the use of physiological indexes to measure subjective experience in VR may present several challenges (see e.g. [19]), for example, the number of uncontrolled, confusing factors in the content of the VR experience, or individual variability of physiological feedback.

\subsection{Theories on the origin of simulator sickness}

Researchers have developed several theories to explain how SS originates. Three theories have previously been referred to as the most widely accepted [27], [28] These theories are the sensory conflict theory[29], the postural instability theory [30], and the eye movements theory [31]. However, other theories have tried to explain the reason behind the adverse reaction of the human physiology towards a specific environment. The theory of Treisman [32], sometimes referred to as evolutionarily or poison theory, tries to explain the evolutionary mechanism that may be related to the phenomena. These theories have often been used in the framework of explaining both MS and SS, as the two phenomena have been shown to have many similarities.

2.1.1 Sensory conflict theory. The probably most cited theory of SS, is the sensory conflict theory, proposed in the work of Reason and Brand [29]. According to this theory, the symptoms of SS derive from a conflict between human sensory systems (visual and vestibular). In a later work [33], the "neural mismatch model" was proposed, suggesting that sickness occurs when sensory information is in conflict with the user's own past experience of a motion environment.

2.1.2 Postural instability theory. The postural instability theory was formulated in the work of Riccio and Stoffregen [34]. According to this theory, MS/SS occur when the user experiences a novel environment, such as in VR, where they have not learned to effectively accommodate and maintain balance [35]. This theory is supported by common reports in sea-travels. Sea travelers get used to the ship's motion, developing what it is referred to as "sea legs". However, when they return onshore, they may experience odd sway when standing or walking, until they do adapt again to walk on settled ground. Such theory is coherent with a study that found that users who are more familiar with interacting in VR environment experience less SS (see [36]).

2.1.3 Eye movement theory. According to the eye movement theory [31], certain stimuli can cause eye movements that result in the stimulation of the vagus nerve, which triggers SS-related symptoms. Ebenholtz proposed in 2001 [37] that certain types of particular eye movements, such as the optokinetic nystagmus and the vestibular ocular response movement, may be connected to MS/SS.

2.1.4 Evolutionary theory. Treisman [32] attempted to explain the reason for MS and SS from an evolutionarily perspective. Treisman [32] suggests that humans are not adapted to new modes of transportation, and that the body responds to sensory conflicts as it would respond if it had ingested a poisonous substance. This theory connects the vomiting response to poison to the same mechanism of nausea in MS and SS ([41,42]).

2.1.5 Other factors. Other factors have been identified and discussed in detail in the published literature, including perception of self-motion (the feelings that the body is moving without being control over the movement) [40], visual display characteristics [41], and technical limitations of the display. The latter theories claim that the available displays are unable to provide a perfect "natural" vision to the user $[5,33,65]$, and the unnatural way that the user's eyes and brains need to behave in modern $3 \mathrm{D}$ environments is to blame for ill-symptoms and fatigue. Due to the emphasis on humancomputer interaction and technology of the present scientific work, 
the technical limitation of VR technology and its possibility to induce SS will be the focus of the discussion of this review.

\section{PROBLEMS WITH DISPLAYS DELIVERING 3D IMAGES}

According to previous studies (see e.g. [44] ), mismatches in the stimuli to vergence and accommodation have a negative health impact on the users, resulting in visual fatigue and discomfort. Because of the problems associated with conventional 3D displays, there have been many attempts to construct displays that minimize the conflict between simulated and focus cues, as well as between vergence and accommodation [45]-[51]. Hoffman et al. [44] proposed a series of experiments to test how humans interact with computerized scenes. They developed a 3D display that presents focus cues that are correct or nearly correct for the depicted scene and used this display to evaluate the influence of focus cues on perceptual distortions, fusion failures, and fatigue. Their experiments show that when focus cues are correct or nearly correct: (1) the time required to identify a stereoscopic stimulus is reduced; (2) stereoacuity in a time-limited task is increased; (3) distortions in perceived depth are reduced; (4) viewer fatigue and discomfort are reduced. Conventional 3D displays are believed to cause fatigue and discomfort, and it has been suggested that these symptoms are caused by differences between the stimuli to vergence and accommodation because such differences require the viewer to uncouple vergence and accommodation [52]-[56]. The evidence offered in support of this hypothesis is that viewers report more fatigue and discomfort when viewing $3 \mathrm{D}$ displays than when viewing $2 \mathrm{D}$ displays. This observation, however, does not prove that vergence-accommodation conflicts cause fatigue and discomfort because there are several other important differences between viewing 2D and 3D displays; these include the eye wear required with 3D displays to separate the two eyes' images, the ghosting or cross-talk from one eye's image to the other's [57] and the perceptual distortions that occur with 3D displays [58] and not with 2D displays [59]. 3D displays present images on one surface, so focus cues-accommodation and blur in the retinal image-specify the depth of the display rather than the depths in the simulated scene. In addition, the vergence stimulus in conventional 3D displays varies depending on where the viewer looks in the simulated scene, but the focal distance remains fixed; the difference in those distances requires the viewer to uncouple vergence and accommodation. Several researchers examined how visual performance, perceived 3D shape, and visual fatigue are affected by the vergence-focal conflicts that arise in conventional 3D displays. Typical 3D displays, like 3D televisions, movies, and AR/VR displays, convey depth information by showing two separate images to our two eyes. As we have discussed, the method can generate most of the depth cues but not the accommodation cue [60]. The simplest way to show an individual image for each eye is to put a display in front of each of the eyes. Such solution is what is adopted by HMDs. To reduce the form factor of the device, micro-displays like small OLED panels are put behind a lens in front of the eyes. The lens generates magnified images of the displays. For 3D screens, where only one display or screen is available contemporarily for both eyes, polarization [61], [62] or color coding [63] technologies can be used to separate the content on the screen to each eye. However, this requires viewers to wear specialized eyeglasses that may cause further discomfort to the users and increase the costs of the technology. To avoid the need for any additional equipment, a lenticular array [64] or a parallax barrier [65] can be placed on top of the display to separate the pixels for each of our eyes. By rendering images with all the psychological depth cues, these methods can drive vergence and create a sense of 3D efficiently. However, since the (magnified) displays are at a fixed distance, human eyes will tend to focus on the same depth regardless of the depth of the virtual objects. As a result, these displays cannot drive accommodation properly and suffer from the vergence accommodation conflict. A link between vergence-accommodation conflict and fatigue/discomfort was proposed and some empirical evidence was found (see e.g. [52], [66]). Psychophysiological studies have been performed to understand the phenomena and eventually find optimal solutions. In a preliminary study, Hagura and Nakajima [67], used a combination of magnetoencephalography (MEG) and functional magnetic resonance imaging (fMRI) to identify the cause of the problems during the vision of stereoscopic images. Frey et al. [68], proposed that the use of electroencephalography signal (EEG) may enable the possibility to create IVT systems which could tune the stereoscopic experience independently to each user.

\subsection{Problems of modern consumer-oriented head-mounted displays}

Modern head mounted displays (HMDs), such as Oculus Rift or HTC Vive, achieve the illusion of depth by presenting each eye with a slightly different image on a flat screen. This means that, no matter how far away an object appears, the eyes remain focused on a fixed point, but they converge on something in the virtual distance. HMDs are in continuous development but are still far from being comfortably worn by any individual in every situation. Such problems arise from both technological and design issues of these devices. According to the remarkable work of Heaney [71], modern Oculus Quest's mechanical inter-pupillary distance (IPD) adjustment makes it most suitable for around $99 \%$ of men and $93 \%$ of women. However, the HMD Rift S, that uses a non-adjustable fixed lenses system, could optimally suit only $46 \%$ of men and $43 \%$ of women. Such problem is probably common for other HMDs, and it is especially severe for those with fixed lenses, such as the Oculus Go. A not optimal adjustment of IPD may produce a sense of discomfort and fatigue in the users (see e.g. [72]). Furthermore, the weight of the devices and the mechanism of fixation of them on the head do cause a sense of discomfort and pressure in the users [73] , worsening the overall quality of the experience. The vergence-accommodation conflict is still an important factor contributing to discomfort in the use of HMDs for VR or for Augment (or Mixed) Reality. Several solutions have been proposed [74]. For these devices, the integration of an eye-tracking system and low-diffraction screens were identified as future research directions. It has been proposed that a combination of various optical designs, as well the use of eye tracking, may be alleviating or eliminating user side-effects from the vergence-accommodation conflict [74]. 


\section{THE USE OF VR IN HEALTH-RELATED APPLICATIONS}

Despite the concerns that the use of computer-generated environments may be detrimental for human health, technological advancements have tried to overcome negative effects related to the use of $\mathrm{VR}$, and to use the technology to help treating health conditions. However, as the technology will be increasingly used in patients affected by medical conditions, the negative side-effects of the use of VR technologies needs more than before to be assessed. Some groups of patients affected by particular conditions may even experience even greater level of discomfort when exposed to VR compared to the healthy population, and therefore the possible benefits from the VR treatments may be impaired or made impossible by the side-effects of the technology. A new wave of psychological research is pioneering VR to diagnose and treat medical conditions from social anxiety [75] to chronic pain [76], [77] and Alzheimer's disease [78]. Riding a wave of interest in mental health tech, companies creating VR content for therapeutic outcomes are receiving a deluge of attention and funding (see e.g. [79], [80]). While VR has been used successfully used to treat post-traumatic stress disorder (PTSD) since the 1990s [81] and anxiety [82], newly developed software may address a much broader range of conditions. Nowadays, health-related VR content is primarily designed to aid exposure therapy, a treatment for anxiety disorders in which patients are exposed to anxiety-inducing stimuli in a safe, controlled environment. For example, someone who fears heights might visit progressively taller buildings under the guidance of their therapist (in vivo exposure), while someone with PTSD might revisit traumatic memories in therapy sessions (imaginary exposure). VR technology gives therapists much more control over the intensity of their patients' experiences, which can lead to better outcomes is such type of treatments [83]. However not all the group of patients may have the same benefits from the use of VR. A study shows that patients with PTSD who also suffer from depression tend to respond much better to VR exposure therapy, compared to other treatment methods [85] This can be explained as imaginary exposure can be a struggle for patients who also have depression since a depressed person may be less able to recall a traumatic memory. It was also shown that virtual reality can be used to create significant distraction for someone who is in physical pain [86]. In addition to potentially providing better outcomes for treatment, VR may aid diagnosis. For example, since the technology can immerse every patient in the same scenario, some researchers believe that VR-based diagnostic testing for conditions like schizophrenia, ADHD and autism could offer more objective results than today's interview-based methods. Further, because VR imitates the patient's everyday environment, it also lets clinicians test symptoms that are usually out of reach, and has been shown [87] that VR tests could enable to diagnose early-stage patients more accurately than report-based methods. Nevertheless, the use of VR could limited its usability in some contexts, as in case of patients that for psychological or physiological conditions show impaired control of movements and balance, as they may easily damage the VR systems and pose their own physical health at risk. Furthermore, those people that are especially sensitive to simulator sickness may see possible benefits of VR-based treatment negatively moderated by the ill-effects of experiencing the VR environments.

\section{CONCLUSION}

The research and development of better immersive visual technologies present several important challenges. Aside from the great implications and benefits that the technology may bring, the psychological and physiological problems that these novel technologies may held them back and discourage their adoptions by a larger public in different areas of use (see e.g. [88], [89]). Limitations of the technology of screens have probably contributed to the negative effect on human health often reported by the users of the technology. However, other theories have been proposed to explain the phenomena of discomfort in VR, specifically, as for example the mismatch between visual and other signals that is typical in many VR environments. Human psychophysiology was shown to be a promising way to assess, and eventually better understand the human-VR interactions and it may contribute to the development of hardware, modality of presentation of the virtual stimuli, or algorithms able to reduce the side effects of the VR experience (as recently attempted in, e.g. [90], [91]). Electroencephalography and especially eye tracking (for its non-invasive nature), have shown to be promising methods to improve human experience in VR. VR technology is already used as a treatment for psychological conditions with promising results. However, such technology and its applications in the clinical settings is too novel to be judged as definitely effective. Future studies should aim to experimentally investigate possibilities and limitations of the use of VR in clinical settings. The question remains whether the benefits obtained from the use of immersive visual technologies are superior to the costs in terms of physical and psychological health. The technology may be mature to be good-enough to be used by a wider public for a number of applications, however there are no studies at the moment that have investigated longitudinally the effect of modern IVTs (e.g. HMDs) on human health on long term use, and, therefore, it is difficult to fully understand the possible negative effects or benefits of such technology. Future technological developments should focus on reducing side effects of the use of the technology, to increase its acceptability as a tool for health promotion.

\section{ACKNOWLEDGMENTS}

This work is funded by EU H2020 MSC-ITN grant agreement No 764951.

\section{REFERENCES}

[1] S. Nichols and H. Patel, "Health and safety implications of virtual reality: a review of empirical evidence," Appl. Ergon., vol. 33, no. 3, pp. 251-271, 2002, doi: 10.1016/s0003-6870(02)00020-0.

[2] J. P. Wann and M. Mon-Williams, "Health issues with virtual reality displays," ACM SIGGRAPH Comput. Graph., vol. 31, no. 2, pp. 53-57, 1997, doi: 10.1145/271283.271307.

[3] M. S. Banks, J. C. A. Read, R. S. Allison, and S. J. Watt, "Stereoscopy and the Human Visual System," SMPTE Motion Imaging f., vol. 121, no. 4, pp. 24-43, 2012, doi: $10.5594 / \mathrm{j} 18173$.

[4] B. T. Backus, M. S. Banks, R. van Ee, and J. A. Crowell, "Horizontal and vertical disparity, eye position, and stereoscopic slant perception," Vision Res., vol. 39, no. 6, pp. 1143-1170, 1999, doi: 10.1016/s0042-6989(98)00139-4.

[5] D. Buckley and J. P. Frisby, "Interaction of stereo, texture and outline cues in the shape perception of three-dimensional ridges," Vision Res., vol. 33, no. 7, pp. 919-933, 1993, doi: 10.1016/0042-6989(93)90075-8.

[6] J. P. Frisby, D. Buckley, and P. A. Duke, "Evidence for Good Recovery of Lengths of Real Objects Seen with Natural Stereo Viewing," Perception, vol. 25, no. 2, pp. 129-154, 1996, doi: 10.1068/p250129. 
[7] J. P. Frisby, D. Buckley, K. A. Wishart, J. Porrill, J. Gårding, and J. E. W. Mayhew, "Interaction of stereo and texture cues in the perception of three-dimensional steps," Vision Res., vol. 35, no. 10, pp. 1463-1472, 1995, doi: 10.1016/0042 6989(95)98726-p.

[8] S. R. Ellis, S. Smith, A. Grunwald, and M. W. McGreevy, "Direction Judgement Error in Computer Generated Displays and Actual Scenes," in Pictorial Communication in Virtual and Real Environments, USA: Taylor \& Francis, Inc., 1991, pp. 504-526.

[9] S. J. Watt, K. Akeley, M. O. Ernst, and M. S. Banks, "Focus cues affect perceived depth," f. Vis., vol. 5, no. 10, p. 7, 2005, doi: 10.1167/5.10.7

[10] S. H. Creem-Regehr, P. Willemsen, A. A. Gooch, and W. B. Thompson, "The Influence of Restricted Viewing Conditions on Egocentric Distance Perception Implications for Real and Virtual Indoor Environments," Perception, vol. 34, no. 2, pp. 191-204, 2005, doi: 10.1068/p5144.

[11] C. S. Sahm, S. H. Creem-Regehr, W. B. Thompson, and P. Willemsen, "Throwing versus walking as indicators of distance perception in similar real and virtual environments," ACM Trans. Appl. Percept., vol. 2, no. 1, pp. 35-45, 2005, doi 10.1145/1048687.1048690.

[12] P. Willemsen, A. A. Gooch, W. B. Thompson, and S. H. Creem-Regehr, "Effects of Stereo Viewing Conditions on Distance Perception in Virtual Environments," Presence Teleoperators Virtual Environ., vol. 17, no. 1, pp. 91-101, 2008 , doi: $10.1162 /$ pres.17.1.91

[13] Z. M. Aghajan, L. Acharya, J. J. Moore, J. D. Cushman, C. Vuong, and M. R. Mehta, "Impaired spatial selectivity and intact phase precession in two-dimensional virtual reality," Nat. Neurosci., vol. 18, no. 1, pp. 121-128, 2014, doi: 10.1038/nn.3884.

[14] J. O. Bailey and J. N. Bailenson, "Immersive Virtual Reality and the Developing Child," Cognitive Development in Digital Contexts. Elsevier, pp. 181-200, 2017, doi: 10.1016/b978-0-12-809481-5.00009-2.

[15] H. Zhang, X. He, and H. Mitri, "Fuzzy comprehensive evaluation of virtual reality mine safety training system," Saf. Sci., vol. 120 , pp. 341-351, 2019, doi: 10.1016/j.ssci.2019.07.009.

[16] K. M. Stanney, R. S. Kennedy, and J. M. Drexler, "Cybersickness is Not Simulator Sickness,” Proc. Hum. Factors Ergon. Soc. Annu. Meet., vol. 41, no. 2, pp. 1138-1142, 1997, doi: 10.1177/107118139704100292.

[17] S. von Mammen, A. Knote, and S. Edenhofer, "Cyber sick but still having fun," Proceedings of the 22nd ACM Conference on Virtual Reality Software and Technology - VRST '16. ACM Press, 2016, doi: 10.1145/2993369.2996349.

[18] T. M. Porcino, E. Clua, D. Trevisan, C. N. Vasconcelos, and L. Valente, "Minimizing cyber sickness in head mounted display systems: Design guidelines and applications," 2017 IEEE 5th International Conference on Serious Games and Applications for Health (SeGAH). IEEE, 2017, doi: 10.1109/segah.2017.7939283.

[19] S. Grassini and K. Laumann, "Questionnaire measures and physiological correlates of presence: A systematic review," Front. Psychol., vol. 11, p. 349, 2020, doi: 10.3389/FPSYG.2020.00349.

[20] R. S. Kennedy, N. E. Lane, K. S. Berbaum, and M. G. Lilienthal, "Simulator Sickness Questionnaire: An Enhanced Method for Quantifying Simulator Sickness," Int. 7 Aviat. Psychol., vol. 3, no. 3, pp. 203-220, 1993, doi: 10.1207/s15327108ijap0303 3 .

[21] S. V. G. Cobb, S. Nichols, A. Ramsey, and J. R. Wilson, "Virtual Reality-Induced Symptoms and Effects (VRISE)," Presence Teleoperators Virtual Environ., vol. 8, no. 2, pp. 169-186, 1999, doi: 10.1162/105474699566152.

[22] B. Keshavarz and H. Hecht, "Validating an Efficient Method to Quantify Motion Sickness," Hum. Factors 7. Hum. Factors Ergon. Soc., vol. 53, no. 4, pp. 415-426, 2011, doi: 10.1177/0018720811403736

[23] B.-C. Min, S.-C. Chung, Y.-K. Min, and K. Sakamoto, "Psychophysiological evaluation of simulator sickness evoked by a graphic simulator," Appl. Ergon., vol. 35, no. 6, pp. 549-556, 2004, doi: 10.1016/j.apergo.2004.06.002.

[24] D. L. Harm, "Motion sickness neurophysiology, physiological correlates, and treatment," in Handbook of Virtual Environments, 2002, pp. 677-702.

[25] M. S. Dennison, A. Z. Wisti, and M. D'Zmura, "Use of physiological signals to predict cybersickness," Displays, vol. 44, pp. 42-52, 2016, doi: 10.1016/j.displa.2016.07.002.

[26] E. Nalivaiko, S. L. Davis, K. L. Blackmore, A. Vakulin, and K. V Nesbitt, "Cybersickness provoked by head-mounted display affects cutaneous vascular tone, heart rate and reaction time," Physiol. Behav., vol. 151, pp. 583-590, 2015, doi 10.1016/j.physbeh.2015.08.043

[27] J. O. Brooks et al., "Simulator sickness during driving simulation studies," Accid. Anal. Prev., vol. 42, no. 3, pp. 788-796, 2010, doi: 10.1016/j.aap.2009.04.013.

[28] N. Dużmańska and A. Strojny Pawełand Strojny, "Can Simulator Sickness Be Avoided? A Review on Temporal Aspects of Simulator Sickness," Front. Psychol., vol. 9, 2018, doi: 10.3389/fpsyg.2018.02132.

[29] J. T. Reason and J. J. Brand, Motion sickness. Academic Press, 1975.

[30] T. A. Stoffregen and G. E. Riccio, "An Ecological Critique of the Sensory Conflict Theory of Motion Sickness," Ecol. Psychol., vol. 3, no. 3, pp. 159-194, 1991, doi: 10.1207/s15326969eco0303_1.

[31] S. M. Ebenholtz, "Motion Sickness and Oculomotor Systems in Virtual Environments," Presence Teleoperators Virtual Environ., vol. 1, no. 3, pp. 302-305, 1992 doi: 10.1162/pres.1992.1.3.302.
[32] M. Treisman, "Motion sickness: an evolutionary hypothesis," Science (80-. )., vol. 197, no. 4302, pp. 493-495, 1977, doi: 10.1126/science.301659.

[33] J. T. Reason, "Motion Sickness Adaptation: A Neural Mismatch Model," f. R. Soc. Med., vol. 71, no. 11, pp. 819-829, 1978, doi: 10.1177/014107687807101109.

[34] G. E. Riccio and T. A. Stoffregen, "An ecological Theory of Motion Sickness and Postural Instability," Ecol. Psychol., vol. 3, no. 3, pp. 195-240, 1991, doi: 10.1207/s15326969eco0303 2.

[35] H. B.-L. Duh, D. E. Parker, and T. A. Furness, "An Independent Visual Background Reduced Simulator Sickness in a Driving Simulator," Presence Teleoperators Virtual Environ., vol. 13, no. 5, pp. 578-588, 2004, doi: 10.1162/1054746042545283.

[36] M. M. Knight and L. L. Arns, "The relationship among age and other factors on incidence of cybersickness in immersive environment users," Proceedings of the $3 r d$ symposium on Applied perception in graphics and visualization - APGV '06. ACM Press, 2006, doi: 10.1145/1140491.1140539.

[37] S. M. Ebenholtz, Oculomotor Systems and Perception. Cambridge University Press, 2001.

[38] K. E. Money and W. S. Myles, "Heavy water nystagmus and effects of alcohol," Nature, vol. 247, no. 5440, pp. 404-405, 1974, doi: 10.1038/247404a0.

[39] K. E. Money and B. S. Cheung, "Another function of the inner ear: facilitation of the emetic response to poisons.," Aviat. Space. Environ. Med., vol. 54, no. 3, pp. 208-11, Mar. 1983.

[40] M. E. McCauley and T. J. Sharkey, "Cybersickness: Perception of Self-Motion in Virtual Environments," Presence Teleoperators Virtual Environ., vol. 1, no. 3, pp. 311-318, 1992, doi: 10.1162/pres.1992.1.3.311.

[41] J. D. Moss and E. R. Muth, "Characteristics of Head-Mounted Displays and Their Effects on Simulator Sickness," Hum. Factors 7. Hum. Factors Ergon. Soc., vol. 53, no. 3, pp. 308-319, 2011, doi: 10.1177/0018720811405196.

[42] T. Shibata, J. Kim, D. M. Hoffman, and M. S. Banks, "The zone of comfort: Predicting visual discomfort with stereo displays," f. Vis., vol. 11, no. 8, p. 11, 2011, doi: $10.1167 / 11.8 .11$

[43] M. S. Banks, J. Kim, and T. Shibata, "Insight into vergence/accommodation mismatch," Head- and Helmet-Mounted Displays XVIII: Design and Applications. SPIE, 2013, doi: 10.1117/12.2019866.

[44] D. M. Hoffman, A. R. Girshick, K. Akeley, and M. S. Banks, "Vergenceaccommodation conflicts hinder visual performance and cause visual fatigue," $\mathrm{F}$. Vis., vol. 8, no. 3, p. 33, 2008, doi: $10.1167 / 8.3 .33$

[45] G. E. Favalora et al., "<title>100-million-voxel volumetric display</title>," Cockpit Displays IX: Displays for Defense Applications. SPIE, 2002, doi: 10.1117/12.480930.

[46] M. Lucente, "Interactive three-dimensional holographic displays," ACM SIGGRAPH Comput. Graph., vol. 31, no. 2, pp. 63-67, 1997, doi: 10.1145/271283.271312.

[47] S. C. McQuaide, E. J. Seibel, R. Burstein, and T. A. Furness, "50.4: Threedimensional Virtual Retinal Display System using a Deformable Membrane Mirror," SID Symp. Dig. Tech. Pap., vol. 33, no. 1, p. 1324, 2002, doi: 10.1889/1.1830190.

[48] B. T. Schowengerdt and E. J. Seibel, "True 3-D scanned voxel displays using single or multiple light sources," f. Soc. Inf. Disp., vol. 14, no. 2, p. 135, 2006, doi: 10.1889/1.2176115.

[49] A. Sullivan, "DepthCube solid-state 3D volumetric display," in Stereoscopic Displays and Virtual Reality Systems XI, 2004, doi: 10.1117/12.527543.

[50] S. Suyama, H. Takada, K. Uehira, S. Sakai, and S. Ohtsuka, "53.3: A New Method for Protruding Apparent 3-D Images in the DFD (Depth-Fused 3-D) Display," SID Symp. Dig. Tech. Pap., vol. 32, no. 1, p. 1300, 2001, doi: 10.1889/1.1831801.

[51] Y. Takaki, "Novel 3D display using an array of LCD panels," Liquid Crystal Materials, Devices, and Applications IX. SPIE, 2003, doi: 10.1117/12.483899.

[52] M. Emoto, T. Niida, and F. Okano, "Repeated Vergence Adaptation Causes the Decline of Visual Functions in Watching Stereoscopic Television," 7. Disp. Technol., vol. 1, no. 2, pp. 328-340, 2005, doi: 10.1109/jdt.2005.858938.

[53] J. Häkkinen, M. Pölönen, J. Takatalo, and G. Nyman, "Simulator sickness in virtual display gaming," Proceedings of the 8th conference on Human-computer interaction with mobile devices and services - MobileHCI '06. ACM Press, 2006, doi: 10.1145/1152215.1152263.

[54] Z. X. Jin, Y. J. Zhang, X. Wang, and T. Plocher, "Evaluating the Usability of an Auto-stereoscopic Display," Lecture Notes in Computer Science. Springer Berlin Heidelberg, pp. 605-614, 2007, doi: 10.1007/978-3-540-73107-8_68.

[55] S. Yano, S. Ide, T. Mitsuhashi, and H. Thwaites, "A study of visual fatigue and visual comfort for 3D HDTV/HDTV images," Displays, vol. 23, no. 4, pp. 191-201, 2002, doi: 10.1016/s0141-9382(02)00038-0.

[56] T. Yamazaki, K. Kamaijo, and S. Fukuzumi, "Quantitative evaluation of visual fatigue encountered in viewing stereoscopic 3D displays: Near-point distance and visual evoked potential study," Proc. Soc. Inf. Disp., vol. 31, pp. 245-7, 1990.

[57] F. L. Kooi and A. Toet, "Visual comfort of binocular and 3D displays," Displays, vol. 25, no. 2-3, pp. 99-108, 2004, doi: 10.1016/j.displa.2004.07.004.

[58] Y. Bereby-Meyer, D. Leiser, and J. Meyer, "Perception of artificial stereoscopic stimuli from an incorrect viewing point," Percept. Psychophys., vol. 61, no. 8, pp. 1555-1563, 1999, doi: 10.3758/bf03213117.

[59] D. Vishwanath, A. R. Girshick, and M. S. Banks, "Why pictures look right when viewed from the wrong place," Nat. Neurosci., vol. 8, no. 10, pp. 1401-1410, 2005, doi: $10.1038 / \mathrm{nn} 1553$. 
[60] O. Cakmakci and J. Rolland, "Head-Worn Displays: A Review," f. Disp. Technol., vol. 2, no. 3, pp. 199-216, 2006, doi: 10.1109/jdt.2006.879846.

[61] H. Kang et al., "3.1: A Novel Polarizer Glasses-type 3D Displays with a Patterned Retarder," SID Symp. Dig. Tech. Pap., vol. 41, no. 1, p. 1, 2010, doi: 10.1889/1.3500405.

[62] S. S. Kim, B. H. You, H. Choi, B. H. Berkeley, D. G. Kim, and N. D. Kim, "31.1: Invited Paper: World's First 240Hz TFT-LCD Technology for Full-HD LCD-TV and Its Application to 3D Display," SID Symp. Dig. Tech. Pap., vol. 40, no. 1, p. 424, 2009, doi: $10.1889 / 1.3256805$

[63] W. Rollmann, "Zwei neue stereoskopische Methoden," Ann. der Phys. und Chemie, vol. 166, no. 9, pp. 186-187, 1853, doi: 10.1002/andp.18531660914.

[64] T. Okoshi, "Three-dimensional displays," Proc. IEEE, vol. 68, no. 5, pp. 548-564, 1980, doi: 10.1109 /proc. 1980.11695 .

[65] J. Y. Son, V. V. Smirnov, H. W. Jeon, Y. J. Choi, and H. S. Lee, "U.S. Patent No. 6,603,504," 2003

[66] S. Yano, M. Emoto, and T. Mitsuhashi, "Two factors in visual fatigue caused by stereoscopic HDTV images," Displays, vol. 25, no. 4, pp. 141-150, 2004, doi: 10.1016/j.displa.2004.09.002.

[67] H. Hagura and M. Nakajima, "Study of asthenopia caused by the viewing of stereoscopic images: measurement by MEG and other devices," Human Vision and Electronic Imaging XI. SPIE, 2006, doi: 10.1117/12.642943.

[68] J. Frey, L. Pommereau, F. Lotte, and M. Hachet, "Assessing the zone of comfort in stereoscopic displays using EEG," Proceedings of the extended abstracts of the 32nd annual ACM conference on Human factors in computing systems - CHI EA '14. ACM Press, 2014, doi: 10.1145/2559206.2581191.

[69] D. Vishwanath and E. Blaser, "Retinal blur and the perception of egocentric distance," 7. Vis., vol. 10, no. 10, p. 26, 2010, doi: 10.1167/10.10.26

[70] M. Zannoli, G. D. Love, R. Narain, and M. S. Banks, "Blur and the perception of depth at occlusions," f. Vis., vol. 16, no. 6, p. 17, 2016, doi: 10.1167/16.6.17.

[71] D. Heaney, "Data Suggests Oculus Rift S IPD Range 'Best' For Just Half Of Adults," 2019. [Online]. Available: https://uploadvr.com/ data-suggests-oculus-rift-s-ipd-range-best-for-around-half- of-adults/.

[72] M. Lambooij, W. IJsselsteijn, M. Fortuin, and I. Heynderickx, "Visual Discomfort and Visual Fatigue of Stereoscopic Displays: A Review," 7. Imaging Sci. Technol, vol. 53, no. 3, p. 30201, 2009, doi: 10.2352/j.imagingsci.technol.2009.53.3.030201.

[73] Y. Yan, K. Chen, Y. Xie, Y. Song, and Y. Liu, "The effects of weight on comfort of virtual reality devices," in Advances in Intelligent Systems and Computing, 2019, vol. 777, pp. 239-248, doi: 10.1007/978-3-319-94706-8_27.

[74] G. Kramida, "Resolving the Vergence-Accommodation Conflict in Head-Mounted Displays," IEEE Trans. Vis. Comput. Graph., vol. 22, no. 7, pp. 1912-1931, 2016 , doi: $10.1109 /$ tvcg.2015.2473855.

[75] P. Anderson, B. O. Rothbaum, and L. F. Hodges, "Virtual reality exposure in the treatment of social anxiety," Cogn. Behav. Pract., vol. 10, no. 3, pp. 240-247, 2003 , doi: 10.1016/s1077-7229(03)80036-6.

[76] D. Gromala, X. Tong, A. Choo, M. Karamnejad, and C. D. Shaw, "The Virtual Meditative Walk," Proceedings of the 33rd Annual ACM Conference on Human Factors in Computing Systems - CHI '15. ACM Press, 2015, doi: 10.1145/2702123.2702344.

[77] T. Jones, T. Moore, and J. Choo, "The Impact of Virtual Reality on Chronic Pain," PLoS One, vol. 11, no. 12, p. e0167523, 2016, doi: 10.1371/journal.pone.0167523.

[78] R. I. García-Betances, M. T. Arredondo Waldmeyer, G. Fico, and M. F. CabreraUmpiérrez, "Corrigendum: A succinct overview of virtual reality technology use in Alzheimer's disease," Front. Aging Neurosci., vol. 7, 2015, doi: 10.3389/fnagi.2015.00235.

[79] S. Lessick and M. Kraft, "Facing reality: the growth of virtual reality and health sciences libraries," F. Med. Libr. Assoc., vol. 105, no. 4, 2017, doi: 10.5195/jmla.2017.329.

[80] B. K. Wiederhold, "Are We Ready for Online Virtual Reality Therapy?," Cyberpsychology, Behav. Soc. Netw., vol. 21, no. 6, pp. 341-342, 2018, doi: 10.1089/cyber.2018.29114.bkw.

[81] L. F. Hodges et al. "A Virtual Environment for the Treatment of Chronic CombatRelated Post-Traumatic Stress Disorder," CyberPsychology Behav., vol. 2, no. 1, pp. 7-14, 1999, doi: 10.1089/cpb.1999.2.7.

[82] B. O. Rothbaum and L. F. Hodges, "The Use of Virtual Reality Exposure in the Treatment of Anxiety Disorders," Behav. Modif., vol. 23, no. 4, pp. 507-525, 1999 , doi: $10.1177 / 0145445599234001$.

[83] A. Rizzo and S. Bouchard, "Virtual Reality for Psychological and Neurocognitive Interventions," Virtual Reality Technologies for Health and Clinical Applications. Springer New York, 2019, doi: 10.1007/978-1-4939-9482-3.

[84] J. Cukor et al., "Virtual Reality Exposure Therapy for Combat-Related PTSD," Posttraumatic Stress Disorder and Related Diseases in Combat Veterans. Springer International Publishing, pp. 69-83, 2015, doi: 10.1007/978-3-319-22985-0_7.

[85] L. Scherer, S, Lucas, GM, Gratch J, Skip Rizzo, A, Morency, "Self-Reported Symptoms of Depression and PTSD Are Associated with Reduced Vowel Space in Screening Interviews," IEEE Trans. Affect. Comput., vol. 7, no. 1, pp. 59-73, 2016, doi: 10.1109/taffc.2015.2440264.

[86] P. Indovina, D. Barone, L. Gallo, A. Chirico, G. De Pietro, and G. Antonio, "Virtual Reality as a Distraction Intervention to Relieve Pain and Distress During Medical Procedures," Clin. F. Pain, p. 1, 2018, doi: 10.1097/ajp.0000000000000599.

[87] O. Kim, Y. Pang, and J.-H. Kim, "The effectiveness of virtual reality for people with mild cognitive impairment or dementia: a meta-analysis," BMC Psychiatry, vol. 19 , no 1,2019 , doi: $10.1186 / \mathrm{s} 12888-019-2180-\mathrm{x}$.

[88] S. Grassini and L. Karin, "Evaluating the use of virtual reality in work safety: literature review," Proceedings of the 30th European safety and reliability conference and the 15th probabilistic safety assessment and management conference, no. 3975, 2020, Research Publishing Services.

[89] S. Grassini, L. Karin, M. R. Skogstad, "The use of virtual reality alone does not promote training performance (but sense of presence does)," Frontiers in Psychology, 2020, vol 11, no 1743, doi: 10.3389/fpsyg.2020.01743.

[90] S. Grassini, K. Laumann, K., V. de Martin Topranin, V, S. Thorp, S, "Evaluating the Efficacy of Multi-Sensory Stimulations on Simulator Sickness and Sense of Presence during HMD-mediated VR Experience," PsyArXiv, October 21, doi:10.31234/osf.io/uv9sd.

[91] S. D'Amour, J.E. Bos, B. Keshavarz, "The efficacy of airflow and seat vibration on reducing visually induced motion sickness," Experimental brain research, 2020, vol 235, no 9, pp. 2811-2820, doi: 10.1007/s00221-017-5009-1. 